\title{
DIFERENÇAS INDIVIDUAIS: TEMPERAMENTO E PERSONALIDADE; IMPORTÂNCIA DA TEORIA ${ }^{1}$
}

\author{
INDIVIDUAL DIFFERENCES: TEMPERAMENT AND \\ PERSONALITY; IMPORTANCE OF THE THEORY
}

\author{
Patrícia do Carmo Pereira ITO ${ }^{1}$ \\ Raquel Souza Lobo Guzzo²
}

\begin{abstract}
RESUMO
Este artigo objetiva apresentar um histórico sobre o estudo do temperamento, bem como o enfoque teórico de diferentes autores sobre sua definição e dimensões. Considerando concepções teóricas distintas, são estabelecidos os principais aspectos que caracterizam o temperamento em todas as teorias. São enfocadas também as principais relações estabelecidas entre temperamento e personalidade e aspectos que os diferenciam.
\end{abstract}

Palavras chave: temperamento, personalidade, diferenças individuais.

\begin{abstract}
This article aims at to present a report on the study of the temperament, as well as the different authors' theoretical focus on her definition and dimensions. Considering different theoretical conceptions, they are established the main aspects that characterize the temperament in all of the theories. They are also focused the main established relationships among temperament and personality and aspects that differentiate them.
\end{abstract}

Key words: temperament, personality, individual differences.

(1) Este texto é parte integrante da dissertação de Mestrado de Patrícia do Carmo Pereira realizada sob orientação de Raquel Souza Lobo Guzzo defendida em fevereiro de 2000, no Instituto de Psicologia e Fonoaudiologia da PUC-Campinas, com o apoio financeiro do CNPq.

Doutoranda em Psicologia pela PUC Campinas, bolsista FAPESP.

Endereço para correspondência: Rua dos Guatás nº 250 apto 12B - Santa Genebra - Campinas/ SP CEP:13081060

Email: paty_ito@directnet.com.br

(2) Professora Titular e Doutora do Instituto de Psicologia e Fonoaudiologia da PUC-Campinas. 
O estudo das diferenças individuais sempre despertou interesse entre teóricos, pesquisadores e leigos. Diferentes dimensões, traços ou características são identificadas nos indivíduos dependendo do enfoque teórico e do interesse.

Já na Grécia antiga, Hipócrates (século IV - V A.C.), o pai da medicina, desenvolveu a teoria dos humores corporais para explicar os estados de saúde e doença. Em sua dissertação intitulada "On the Nature Man", deduz dos quatro elementos primários do universo, terra, ar, fogo e água, quatro qualidades: calor, frio, úmido e seco, as quais foram relacionadas à quatro humores corporais: sangue, fleuma, bile branca e bile negra. O equilíbrio adequado entre estes humores determinaria a saúde, e o desequilíbrio causaria a doença (Strelau, 1998).

Baseado na teoria de Hipócrates, Galeno desenvolveu a primeira tipologia do temperamento, descrita em sua monografia "De Temperamentis", onde distinguiu e descreveu nove temperamentos: quatro temperamentos primários relacionados à dominância de uma das quatro qualidades descritas por Hipócrates; quatro temperamentos secundários, derivados do pareamento entre as qualidades, e um temperamento resultado da mistura estável das quatro qualidades, considerado como temperamento ideal (Strelau, 1998).

Os quatro temperamentos primários estabelecidos e descritos por Galeno, são conhecidos entre teóricos e leigos, sendo nomeados de acordo com os humores predominantes no corpo: 1) tipo sangüíneo, caracterizado por indivíduos atléticos e vigorosos, nos quais o humor corporal predominante era o sangue; 2) tipo colérico, indivíduos facilmente irritáveis, nos quais predominava a bile amarela; 3) tipo melancólico, indivíduos tristes e melancólicos que exibiam excesso de bile negra; e 4) tipo fleumático, indivíduos cronicamente cansados e lentos em seus movimentos, que possuíam excesso de fleuma (Aiken, 1991).

Esta tipologia do temperamento exerceu forte influência em teóricos da Alemanha, Estados Unidos, França, Itália e Polônia, postulando desde períodos tão antigos que as diferenças no comportamento poderiam ser explicadas por mecanismos fisiológicos e bioquímicos.

Em fins do século XVIII e meados do século XIX, é possível observar influências da tipologia estabelecida pelos antigos gregos, nas teorias de temperamento de estudiosos alemães como Immanuel Kant e Wilhelm Wundt.

Immanuel Kant, em 1798, publicou sua "Anthropology", na qual descrevia sua teoria de temperamento. Considerava este constructo como um fenômeno psicológico, compreendido por traços psíquicos determinados pela composição do sangue, que estaria relacionada a facilidade ou dificuldade da coagulação sangüínea e também sua temperatura. Kant distinguiu quatro tipos de temperamento considerando a composição sangüínea e usando critérios de energia de vida, que oscilam da excitabilidade à sonolência, além de características do comportamento dominante como emoção versus ação: 1) sangüíneo, caracterizado pela força, rapidez e emoções superficiais; 2) melancólico, designado pelas emoções intensas e vagarosidade das ações; 3) colérico, rapidez e impetuosidade no agir; e 4) fleumático, caracterizado pela ausência de reações emocionais e vagarosidade no agir (Strelau, 1998).

Já Wilhelm Wundt, estudando emoções e tempo de reação em seu laboratório, deparouse com a constatação da existência de diferenças individuais nas reações emocionais, denominadas temperamento. Segundo este autor, temperamento define-se como disposições aplicadas às direções das emoções. Partindo de dois fatores emocionais, força e velocidade da mudança, Wundt 
distinguiu quatro tipos de temperamento: 1 ) coléricos e melancólicos, caracterizados pela força das emoções; 2) sangüíneos e fleumáticos, designados pela fraca emoção; 3) sangüíneos e coléricos, caracterizados pelas mudanças emocionais rápidas, e 4) melancólicos e fleumáticos, caracterizados por mudanças emocionais lentas (Strelau, 1998).

Apesar dos estudos de longa data, foi no início do século XX que psiquiatras e psicólogos começaram a desenvolver estudos mais efetivos sobre temperamento, alguns como Carl Gustav Jung e Alfred Adler, desenvolveram teorias mais especulativas, outros como Gerard Heymans, Ernst Kretschmer e Ivan Pavlov baseados em diferentes abordagens teóricas e provenientes de diferentes países, conduziram estudos mais empíricos.

As formulações teóricas mais especulativas que influenciaram as teorias mais contemporâneas de personalidade eram as de:

1. Carl Gustav Jung postulava que os indivíduos eram caracterizados por dois tipos de atitude, a extroversão e a introversão, as quais eram de origem biológica e refletiam a direção em que a energia psíquica era expressa. A extroversão, segundo ele, era governada por expectativas e necessidades sociais, estando orientada para a adaptação e reações exteriores, enquanto a introversão teria sua energia dirigida para os estados subjetivos e processos psíquicos.

Os dois tipos de atitudes tornaram-se as mais populares dimensões de personalidade $e$ temperamento, sendo incorporadas em teorias de personalidade, como as de Cattell, Guilford e Hans J. Eysenck e pesquisas de temperamento, como as de Kagan e seu temperamento inibido e desinibido (Strelau, 1998).

2. Adler postulava a existência de quatro tipos de temperamento, os quais também foram baseados na tipologia de Galeno, e definidos de acordo com o interesse social e nível de energia manifesto pelos indivíduos: a) tipo governante (ruling type)- caracterizado por indivíduos com certo nível de agressividade, tiranos, enérgicos e dominantes, estando os mesmos relacionados ao tipo colérico por apresentarem características temperamentais semelhantes às encontradas neste tipo; b) tipo dependente (leaning type) - pessoas sensíveis, que desenvolvem em torno de si uma concha para protegerem-se dos eventos externos, possuem baixos níveis de energia e são caracterizados como dependentes, constituindo o tipo fleumático, exemplificado por indivíduos cronicamente cansados e pouco dispostos; c) tipo de evitação (avoiding type) indivíduos que apresentam como padrão de vida o afastamento do contato direto com pessoas e circunstâncias, mantêm baixos níveis de energia, e são caracterizados pelo tipo melancólico, predominantemente tristes; e d) tipo socialmente útil (socially useful type)pessoas saudáveis, que apresentam interesse social e energia, estando relacionadas ao tipo sangüíneo, caracterizando indivíduos atléticos e vigorosos (Boeree, 1998b).

Entre os pesquisadores empiricistas do início do século, é possível destacar Heymans, Kretschmer e Pavlov:

1. Gerard Heymans, da Holanda, o qual empreendeu a primeira pesquisa sistemática de temperamento usando a abordagem experimental, psicométrica e biográfica. Tinha como objetivo descrever as dimensões básicas do temperamento e determinar em que grau hereditariedade e ambiente contribuem para o desenvolvimento dos traços temperamentais. A pesquisa de Heymans, realizada em 1905 contou com a colaboração de Wiersma. Consistiu na distribuição de um questionário de 90 itens para mais de 3000 médicos, no qual thes era solicitado que avaliassem o comportamento e características psíquicas de famílias (incluindo pais, mães e filhos) que conhecessem bem. Retornaram os questionários de mais de 400 médicos, 
totalizando dados de 437 famílias, somando 2415 sujeitos (437 pais, 437 mães e 1541 crianças). Heymans distinguiu três dimensões de temperamento baseando-se nas considerações teóricas de Kant, Gross e Wundt, além de dados de seu estudo empírico sobre percepção sensorial: a) emocionalidade, relacionada a sensibilidade ou excitabilidade das emoções; b) atividade, diz respeito ao comportamento operante; e c) funcionamento primário e secundário, que refere-se ao aspecto temporal do comportamento e do processo psíquico. Estas dimensões, consideradas também em seus pólos opostos, deram origem à tipologia de temperamento de Heymans, composta por oito tipos de temperamento: amorfo, apático, nervoso, sentimental, sangüíneo, fleumático, colérico e apaixonado (Strelau, 1994, 1998).

Heymans é considerado um teórico importante na área de temperamento, pois além de desenvolver o primeiro estudo empírico, suas dimensões de temperamento, principalmente atividade e emocionalidade, ganharam alta popularidade e estão incluídas na estrutura de temperamento de muitos teóricos contemporâneos, embora com significados diferentes. Sua notoriedade também é devida, ao envolvimento de famílias (pai, mãe e filhos) inteiras em sua pesquisa, que possibilitaram informações sobre a hereditariedade do temperamento. Seu estudo é considerado precursor das pesquisas em genética comportamental, na área do temperamento e personalidade (Strelau, 1998).

2. Ernst Kretschmer, psiquiatra alemão, desenvolveu sua teoria de temperamento baseando-se em estudos e pesquisas, na qual correlacionava biotipo físico, propensão a certo tipo de doença e temperamento. A partir de seus dados, distinguiu três tipos temperamentais: a) esquizotímico, caracterizado por indivíduos astênicos, reservados, apresentando emoções que oscilam da irritabilidade a indiferença, rígidos nos hábitos e atitudes, com dificuldades de adaptação e propensos à esquizofrenia; b) ciclotímico, caracterizado por indivíduos rotundos, com emoções que variavam da alegria a tristeza, facilidade de estabelecer contato com o ambiente, realísticos em suas visões e propensos ao distúrbio maníaco depressivo; c) isotímico, indivíduos atléticos, tranqüilos, com pouca sensibilidade, modestos nos gestos e imitações, com dificuldade de adaptação ao seu ambiente, propensos à epilepsia. Esta tipologia constitucional ficou conhecida na Europa, principalmente na década 30 (Strelau, 1998).

3. Ivan P. Pavlov, da Rússia, começou a desenvolver seus estudos no início do século $\mathrm{XX}$, primeiro teórico a realizar estudos sobre o temperamento em laboratório e a propor uma tipologia do sistema nervoso, explicando as diferenças individuais como respostas de processos de condicionamento. Este autor, em seus estudos experimentais com cães, distinguia quatro tipos de sistema nervoso (sistema nervoso forte, equilibrado e móvel; forte equilibrado e inerte; forte e não equilibrado e sistema nervoso fraco), os quais resultariam de diferentes configurações das quatro propriedades fundamentais do sistema nervoso central: força de excitação, força de inibição, equilíbrio e mobilidade do processo nervoso (Strelau, 1997; Strelau, Angleitner \& Newberry, 1999).

Pavlov (apud Strelau, 1998) acreditava que o tipo de sistema nervoso era uma característica inata e relativamente imune às influências ambientais, e que os quatro tipos de sistema nervoso poderiam ser relacionados aos tipos clássicos de temperamento propostos na tipologia de Hipócrates-Galeno, conforme esquema abaixo:

Embora a tipologia de sistema nervoso de Pavlov tenha sido estabelecida em experimentos e observações de cachorros, ele acreditava que estes tipos poderiam ser estendidos ao homem. De acordo com ele, os tipos de sistema nervoso estabelecido em animais, quando referentes ao homem, são chamados de temperamento (Strelau, 1998, Strelau, Angleitner \& Newberry, 1999). 




Figura 1. Tipos de sistema nervoso relacionados aos tipos clássicos de temperamento da tipologia Hipócrates - Galeno

Pavlov apresentou o vínculo entre características temperamentais e tipos de sistema nervoso. Utilizando o paradigma do condicionamento reflexo, introduziu medidas objetivas e psicofisiológicas em estudos experimentais de temperamento, proporcionando a primeira evidência de que as diferenças individuais na velocidade e na estabilidade dos condicionamentos reflexos estariam relacionadas ao temperamento. Pavlov reconheceu a significância funcional do temperamento e o papel das propriedades do sistema nervoso central na adaptação do indivíduo ao ambiente. Seus constructos influenciaram as teorias de personalidade e os estudos sobre temperamento mais contemporâneos (Strelau, 1997).

Em meados de 1950, torna-se crescente o interesse pelo temperamento. Começam a surgir os estudos contemporâneos, cujos principais representantes são Hans J. Eysenck do Maudsley Hospital, Universidade de Londres; Boris M. Teplov do Instituto de Psicologia, da Academia de Ciências Pedagógicas em Moscou; e Alexander Thomas e Stella Chess, psiquiatras do New York University Medical Center (Strelau, 1998).

1. Eysenck, eminente estudioso no campo da personalidade, considerava as diferenças individuais como sendo produzidas pela herança genética, sendo que seu principal foco de interesse era o temperamento (Boeree, 1998a). Este teórico postulava que o temperamento tem origem biológica, e seus traços são universais. A partir de exaustivos estudos de análise fatorial conduzidos durante várias décadas, com várias populações, assim como resultados de técnicas psicométricas e experimentações de laboratório, concluiu que a estrutura de temperamento consistia de três fatores básicos: psicoticismo $(P)$, extroversão (E) e neuroticismo $(\mathrm{N})$, conhecidos entre teóricos da personalidade como PEN ( (Strelau, 1998).

2. Teplov e seus colaboradores, influenciados pelas idéias de Pavlov, consideravam o temperamento como a expressão comportamental e psicológica das propriedades dos sistema nervoso central, estando relacionado às características dinâmicas do comportamento expresso nas diferenças individuais na velocidade e intensidade das reações. Dentro desta perspectiva, uma visão mais elaborada do temperamento foi apresentada por Nebylistsyn em seu escrito publicado na "Enciclopédia Pedagógica", segundo o qual temperamento era uma característica individual, expressa em aspectos do comportamento tais como: tempo, velocidade, e ritmo, consistindo em três componentes maiores: atividade, movimento e emocionalidade. De acordo com Teplov e Nebylistsyn, traços de temperamento não são suscetíveis à mudanças e o fato do 
temperamento ser considerado inato, mas não necessariamente herdado, era usado por Teplov e seus colaboradores como principal argumento em favor da estabilidade temperamental (Strelau, 1998).

3. Thomas e Chess realizaram importante estudo longitudinal, e são reconhecidos como importantes teóricos do temperamento. Influenciando diversos estudiosos, eles igualavam este constructo a idéia de estilo comportamental. Para estes autores: a) o temperamento é um atributo psicológico que interage com outros atributos, mas é independente dos mesmos; b) apesar do temperamento interagir com outros atributos é importante que cada um deles seja identificado separadamente, devendo-se analisar a situação em que o comportamento ocorre; c) o temperamento pode ser considerado um atributo mediador entre influência do ambiente e a estrutura psicológica do indivíduo (Thomas, Chess e Korn, 1982).

Em 1956, Thomas e Chess iniciaram importante estudo, que teve continuidade por mais de 30 anos, sendo conhecido na literatura como "New York Longitudinal Study" (NYLS), o qual envolvia bebês a partir dos dois ou três meses de vida. Através deste estudo longitudinal distinguiram nove categorias de temperamento: ritmicidade de funções biológicas; nível de atividade; aproximação ou afastamento frente a novos estímulos; adaptabilidade; limite sensorial; qualidade predominante de humor; intensidade de expressões de humor; distração e persistência. Análises qualitativas da significância funcional das nove categorias de temperamento, apoiadas pela análise fatorial, levaram os autores a distinguir três constelações temperamentais: crianças de temperamento fácil, de temperamento difícil e de aquecimento lento. Inerente a estas constelações temperamentais, os autores introduziram o conceito de "goodness of fit" (adaptação excelente), em cuja condição as capacidades individuais, temperamento e outras características individuais estão de acordo com as oportunidades, demandas e expectativas do ambiente, especialmente de pais, professores, e pares (Chess e Thomas, 1991).

As conceitualizações de Eysenck, Thomas e Chess e Teplov e Nebyllitsyn estimularam muitos pesquisadores a desenvolverem novas teorias sobre o temperamento, ou ainda, modificar as existentes. Entre estes novos pesquisadores, é possível destacar as contribuições teóricas de Goldsmith e colaboradores (1987), Buss \& Plomin (Buss, 1995), Rothbart (1986a, 1986b), Strelau (1991, 1994, 1995, 1998) e Lerner e Windle (Lerner, Lerner, Windle, Hooker, Lerner e East, 1986).

1. Goldsmith e seus colaboradores (Goldsmith e colaboradores, 1987) caracterizam temperamento como "diferenças individuais na probabilidade de experienciar e expressar as emoções primárias" (p.520). O temperamento é "emocional em sua natureza, pertence às diferenças individuais e se refere mais as tendências de comportamento que as ocorrências atuais de comportamento emocional" (p.511). Os autores consideram o temperamento como um fator relativamente estável, que se apresenta independente de outros fatores, e que as dimensões temperamentais (representadas pelas emoções primárias: raiva, medo, alegria, prazer, interesse e atividade motora, esta última refletindo ativabilidade emocional) formam a base emocional do desenvolvimento da personalidade, a qual é composta por diversos fatores que interagem com as características temperamentais. Também são considerados complementares ao temperamento os aspectos receptivos, a habilidade de reconhecer e decodificar as expressões emocionais de outros.

2. Buss e Plomin definem temperamento como traços de personalidade herdados que aparecem durante os primeiros dois anos de vida e permanecem como componentes 
básicos, sendo compostos por quatro categorias: emotividade, atividade, sociabilidade e impulsividade (Buss, 1995).

3. Rothbart (1986a, 1986b) define temperamento como "diferenças individuais biologicamente baseadas na reatividade e auto - regulação", sendo que a reatividade se refere à intensidade e a aspectos temporais dos comportamentos ligados ao sistema nervoso central, autonômo e endócrino; e a autoregulação representa os processos que modulam as reações, incluindo comportamentos como aproximação, imitação e atenção. Para a autora é clara a distinção entre temperamento e personalidade. $\mathrm{O}$ temperamento seria considerado como a base biológica para a estruturação da personalidade e um dos fatores que influenciam o comportamento, enquanto a personalidade seria um termo mais amplo que inclui outras estruturas importantes além dessas, tais como as estruturas cognitivas, e auto conceito. A autora propõe algumas dimensões que podem sofrer alterações ao longo do tempo, e são consideradas como elementos de temperamento: a) reatividade negativa (aversão à aproximação e expressão de sentimentos negativos); b) reatividade positiva (aproximação e expressão de sentimentos positivos); c) inibição comportamental para estímulos novos e intensos e d) capacidade de fixar a atenção.

4. Windle e Lerner (Lerner, Lerner, Windle, Hooker, Lerner e East, 1986) consideram que as características temperamentais podem influenciar o tipo de interação que será estabelecido entre a pessoa e seu ambiente. Estes autores (Windle e Lerner, 1984) propõem em suas investigações, a avaliação do temperamento através das dimensões: aproximação - retração; flexibilidade - rigidez, nível de atividade no sono, ritmicidade, humor e persistência.

5. Strelau $(1991,1994,1998)$ tem seus pressupostos teóricos sobre temperamento baseados nas concepções de funcionamento do sistema nervoso de Pavlov, e em pesquisas e teorias desenvolvidas no período de 1950 e 1960 na Europa Ocidental e nos Estados Unidos, as quais deram origem à Teoria Regulativa do Temperamento - RTT. O autor considera que:

"o temperamento se refere a traços básicos, relativamente estáveis, expressos principalmente nas características formais de reações e comportamento. Estes traços estariam presentes desde o início da vida na criança. Primariamente determinado por mecanismos de origem biológica, o temperamento estaria sujeito a mudanças causadas pela maturação e pela interação indivíduo - genótipo específico - ambiente" (Strelau, 1998, p.165).

Nesta perspectiva, a estrutura do temperamento seria descrita a partir de seis traços: ativação, perseveração, sensibilidade sensorial, reatividade emocional, resistência e atividade.

Como é possível perceber, os estudos sobre temperamento têm sido realizados desde há muito tempo, desenvolvidos por estudiosos de diferentes abordagens e envolvendo os mais diferentes métodos de investigação. Ainda assim, um consenso sobre sua definição e dimensões parece estar longe de acontecer.

Apesar de vários estudiosos terem sido influenciados pela tipologia de Galeno, os pesquisadores contemporâneos basearam suas teorias em diferentes abordagens, enfatizando diferentes aspectos. Dependendo da abordagem teórica adotado pelo autor, a definição do temperamento e suas dimensões variam, assim como seus instrumentos de medida deste fenômeno ou constructo.

$\mathrm{Na}$ tentativa de chegar a um consenso sobre a definição do temperamento, Goldsmith e Rieser-Danner (1986) levantaram os principais pontos de divergência e acordo entre as principais teorias.

No que se refere às controvérsias teóricas, estas estão relacionadas a: 1) número 
diferenciado de dimensões do temperamento; 2) diferentes ênfases dadas ao fator biológico; 3) função da motivação no temperamento; 4) definições de temperamento, que em alguns casos dizem respeito ao aspecto comportamental, mas em outros se referem ao aspecto psicofisiológico; 5) alguns teóricos enfatizam a regulação e o controle de componentes do comportamento como aspecto do temperamento, enquanto outros se referem a estilos de comportamento; 6) diferentes concepções relacionadas às influências do contexto e das relações interpessoais no temperamento; e 7) diferentes limites são estabelecidos entre personalidade e temperamento (Goldsmith e Rieser-Danner, 1986).

Os pontos de acordo entre os diferentes teóricos referem-se a: 1) temperamento como dimensões gerais de comportamento, as quais caracterizam diferenças individuais, representando padrões universais de desenvolvimento; 2) características temperamentais aparecem durante a infância e representam parte da fundamentação da personalidade posterior; 3) as dimensões temperamentais são relativamente estáveis ao longo do tempo; 4) os traços temperamentais apresentam substrato biológico; e 5) a expressão das características temperamentais podem sofrer influências de fatores do contexto (Goldsmith e Rieser-Danner, 1986).

Um outro aspecto controverso relacionado ao temperamento diz respeito a sua diferenciação de personalidade. Segundo Teiglasi (1995), o contraste entre estes conceitos é obscuro, pois eles apresentam um vocabulário descritivo comum, chegando a ocorrer em alguns casos a superposição de conceitos, uma outra dificuldade está relacionada a carência de dados empíricos capazes de diferenciar estes conceitos com base nos fatores biológicos. Para Strelau (apud Hofstee, 1991) existem três maneiras pelas quais temperamento e personalidade relacionam-se nas diferentes abordagens teóricas: 1) temperamento pode ser considerado um dos elementos da personalidade, 2) pode ser considerado sinônimo de personalidade, ou 3) um fenômeno não pertencente a personalidade.

Este mesmo autor estabelece ainda cinco diferenças entre temperamento e personalidade, as quais dizem respeito à: 1) o temperamento é biologicamente determinado, e a personalidade é um produto do ambiente social; 2) os traços temperamentais podem ser identificados desde cedo na criança, e a personalidade é compartilhada em períodos posteriores do desenvolvimento; 3) diferenças individuais nos traços de temperamento, como ansiedade, extroversão - introversão e busca de estimulação, são também observadas em animais, enquanto a personalidade é prerrogativa do humano; 4) o temperamento apresenta aspectos estilísticos, se referindo a características formais de comportamento, já a personalidade contém aspectos relativos a conteúdos do comportamento; 5) ao contrário de temperamento, que se refere principalmente a traços ou mecanismos, a personalidade está relacionada ao funcionamento integrativo do comportamento humano (Strelau, 1998).

Para Strelau (1991, 1994, 1998) a personalidade seria um conceito mais amplo que temperamento, que incluiria o próprio temperamento e outras características que são geralmente determinadas pelo ambiente social, modeladas pelos estágios de desenvolvimento do indivíduo e envolveria ainda fenômenos como motivação, valores e interesses.

A este respeito, na última década, ganhou destaque a discussão realizada sobre a relação dos fatores de personalidade do "Big Five", considerado a taxonomia das características de personalidade, e o temperamento. Vários fatores relacionados pelo "Big Five" (extroversão, agradabilidade, concienciosidade, estabilidade emocional e cultura) são também relacionados pelos teóricos como dimensões ou traços de temperamento. O 
primeiro estágio de pesquisas relacionadas a este aspecto, consiste principalmente em especulações e hipóteses. Teóricos do desenvolvimento consideram que as características temperamentais infantis podem ser precursoras dos fatores do "Big Five" encontrados em adolescentes e adultos, porém são necessários maiores estudos para comprovação deste fato (Strelau, 1998; Strelau \& Angleitner, 1991).

Tópicos relacionados ao temperamento têm recebido especial atenção no meio científico internacional. Um aspecto importante a ser considerado no estudo deste constructo é o de que seja adotada uma abordagem teórica consistente e que fundamente instrumentos com reconhecidas qualidades psicométricas, para que estes possam ser utilizados na avaliação de indivíduos.

Tal cuidado e interesse deve-se ao fato de que o temperamento influencia o desenvolvimento psicológico, ele é o resultado de interações entre temperamento, outras características do indivíduo e o ambiente social (Strelau e Angleitner, 1991).

Neste sentido a avaliação do temperamento apresenta-se como tópico importante para o tratamento de desordens psicológicas e problemas psicossociais e, principalmente, para ações preventivas.

\section{REFERÊNCIAS}

AIKEN, L.R. (1991). Psychological testing and assessment $\left(7^{\text {th }} \mathrm{ed}\right)$. Massachusetts: Allyn and Bacon.

BOEREE, C.G. (1998a). Hans Eysenck and other temperament theorists. [On line] http:/ /www.ship.edu/ cgboeree/eysenck.html.

BOEREE, C.G. (1998b). Personality theories: Alfred Adler. [On line] http://www.ship.edu/ $\sim$ cgboeree/adler.html.
BUSS, A.H. (1995). Personality - temperament, social behavior, and the self. Massachusets: Allyn and Bacon.

CHESS, S., \& Thomas, A. (1991). Temperament and the concept of goodness of fit. In J. Strelau, \& A. Angleitner (Eds), Explorations in Temperament (pp. 15-28). New York: Plenum Press.

GOLDSMITH, H.H., Buss, A.H., Plomin, R., Rothbart, M.K., Chess, S. Thomas, A., Hinde, R.A., \& Mccall, R.B. (1987). Rountdtable: What is temperament? Four approaches. Child Development, 58, 505-529.

GOLDSMITH, H.H., \& Rieser-Danner, L.A. (1986). Variation among temperament theories and validation studies of temperament assessment. In G.A. Kohnstamm (Ed.), Temperament discussed - temperament and development in infancy and childhood, (pp. 1-10). Lisse: Swets \& Zeitlinger.

HOFSTEE, W.K.B. (1991). The conceptsof personality and temperament. In J. Strelau, \& A. Angleitner (Eds), Explorations in temperament - international perspective on theory and measurement. (pp. 177 - 188). New York: Plenum Press.

LERNER, R.M., Lerner, J.V., Windle, M., Hooker, K., Lerner, K. \& East, P.L. (1986). Children and adolescents in their contexts: tests of a goodness of fit model. In R. Plomin, \& J. Dunn (Eds), The study of temperament: changes, continuities and challenges. Erlbaum: Hillsdale.

ROTHBART, M.K. (1986a). A psychobiological approach to the study of temperament. In G.A. Kohnstamm (Ed.), Temperament discussed - temperament and development in infancy and childhood (pp. 63-72). Lisse: Swets \& Zeitlinger.

ROTHBARTH, M.K. (1986b). Longitudinal observation of infant temperament. Development Psychology, 22 (3), 356-365. 
STRELAU, J. (1991). Temperament and Giftedness in Children and Adolescents. Conferência apresentada na "Ninth world conference on gifted and talented children" - Hage Netherlands.

STRELAU, J. (1994). The concepts of arousal and arousability as used in temperament Studies. In J.E. Bates, \& T.D. Wachs, (Eds.), Temperament individual differences at the interface of biology and behavior (pp. 117-141). Washington: American Psychological Association.

STRELAU, J. (1995). The regulative theory of temperament: current status. Paper presented at the 7th Meeting of International Society for the Study of Individual Differences, Warsaw, Poland.

STRELAU, J. (1997). The contribution of Pavlov's typology of CNS properties to personality research. European Psychologist, 2 (2), 125-138.

Strelau, J. (1998). Temperament: A Psychological Perspective. New York: Plenum.
STRELAU, J. \& Angleitner, A. (1991) Temperament research: some divergences and similarities. In J. Strelau, \& A. Angleitner (Eds), Explorations in temperament - international perspective on theory and measurement (pp. 1-12). New York: Plenum.

STRELAU, J., Angleitner, A., \& Newberry, B.H (1999). The Pavlovian Temperament Survey (PTS): An International Handbook. Seattle: Hogrefe \& Huber Publishers.

TEIGLASI, H. (1995). Assessment of temperament. Eric Digest [on line] http:// www.ed.gov/databases/eric-digest/ ed389963.html.

THOMAS, A., Chess, S., e Korn, S.J. (1982) The reality of diffcult temperament. Merril Palmer Quartely, 28 (1), 1-20.

WINDLE, M. \& Lerner, R.M. (1984). The role of temperament in dating relationships among young adults. Merril - Palmer Quarterly, 30 (2), 163-175. 